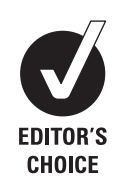

CHOICE

\title{
Early structural changes in cartilage and bone are required for the attachment and invasion of inflamed synovial tissue during destructive inflammatory arthritis
}

\author{
Adelheid Korb-Pap, ${ }^{* 1,2}$ Athanasios Stratis, ${ }^{* 3}$ Katja Mühlenberg, ${ }^{3}$ Birgit Niederreiter, ${ }^{1}$ \\ Silvia Hayer, ${ }^{1}$ Frank Echtermeyer, ${ }^{4}$ Richard Stange, ${ }^{3}$ Jochen Zwerina, ${ }^{5}$ Thomas Pap, ${ }^{3}$ \\ Hermann Pavenstädt, ${ }^{2}$ Georg Schett, ${ }^{6}$ Josef S Smolen, ${ }^{1}$ Kurt Redlich ${ }^{1}$
}

\begin{abstract}
- An additional figure is published online only. To view the file please visit the journal online (http://ard.bmj.com/ content/71/6.toc).
\end{abstract}

${ }^{1}$ Division of Rheumatology, Medical University Vienna, Vienna, Austria

${ }^{2}$ Department of Internal Medicine D, Nephrology and Rheumatology, University Hospital Munster, Muenster, Germany

${ }^{3}$ Institute of Experimental Musculoskeletal Medicine, University Hospital Munster, Muenster, Germany ${ }^{4}$ Department of Experimental Anaesthesia, University Hospital Hanover, Hanover, Germany ${ }^{5} 4$ th Medical Department, Hanusch Hospital, Vienna, Austria

${ }^{6}$ Department of Internal Medicine 3, University of Erlangen, Erlangen, Germany

\section{Correspondence to}

Kurt Redlich, Division of Rheumatology, Department of Internal Medicine III, Medical University Vienna, Währinger Guertel 18-20, A-1090 Austria; kurt.redlich@meduniwien.ac.at

*AK-P and AS contributed equally to this work.

Received 30 June 2011 Accepted 4 December 2011 Published Online First 18 January 2012

\begin{abstract}
Objective To elucidate the mechanisms involved in cartilage damage in an experimental model of rheumatoid arthritis (RA) by specifically addressing the time course of extracellular matrix degradation and the contribution of cell-matrix interactions for initiation and perpetuation of this process.
\end{abstract}

Methods The human tumour necrosis factor (TNF) transgenic (hTNFtg) mouse model of RA was used to analyse the time course of pannus attachment to the cartilage and cartilage destruction, respectively, and crossed hTNFtg mice with interleukin (IL)-1 $1^{-/-}$animals were used to investigate the role of IL-1 on these TNF-induced mechanisms in vivo. In addition, an in vitro attachment assay using synovial fibroblasts (SFs) from hTNFtg mice and freshly isolated articular cartilage was used to determine the role of proteoglycan loss in attachment of SFs and the role of the transmembrane heparan sulfate proteoglycan syndecan-4.

Results In vivo analyses of hTNFtg mice showed that proteoglycan loss induced by IL-1 precedes and constitutes an important prerequisite for these processes as, in hTNFtg mice, IL-1 deficiency protected from the loss of cartilage proteoglycans and almost completely prevented the attachment and subsequent invasion of inflamed synovial tissue into cartilage. In vitro studies confirmed that loss of cartilage proteoglycans is required for attachment of SFs and that syndecan-4 is prominently involved in SF attachment and activation.

Conclusions The results of this study suggest that the loss of cartilage proteoglycans is an early event in the course of destructive arthritis that facilitates the attachment of the inflamed synovial membrane and also initiates matrix degradation and inflammation through cell-matrix interactions.

\section{INTRODUCTION}

Rheumatoid arthritis (RA) is a chronic and debilitating disease of the joints characterised by synovial inflammation and progressive destruction of articular cartilage and bone. ${ }^{12}$ Despite a growing understanding of the clinical course of RA, major parts of its pathogenesis are still poorly understood. This is particularly true for early phases of the disease when the process of destruction is initiated. It is generally accepted that biochemical and cellular changes precede clinical manifestations of RA. Moreover, joint destruction starts before it can be visualised even by most advanced techniques. The specific sequences of cellular events and interactions leading to progressive destruction of articular structures have therefore not been clarified. Specifically, it has been a matter of intense debate whether (1) changes of the articular cartilage can be found prior to the attachment of inflamed synovial tissue to the cartilage; (2) cartilage damage leads to subsequent interaction of the cartilage matrix with the inflamed synovium; (3) this interaction is essential for the progression of disease; and (4) cartilage damage precedes bony erosions or vice versa.

Although an early in vitro study suggested that damaged cartilage facilitates the attachment of cells, especially of monocytes, ${ }^{3}$ the in vivo relevance of these findings as well as their implications on the invasion of synovial tissue and the course of joint destruction remains elusive.

On the other hand, there is a bulk of evidence that the primary attachment of synovial cells, particularly synovial fibroblasts (SFs), to matrix components such as collagens and proteogylcans contributes to activation of signalling pathways, triggering their invasive behaviour. ${ }^{4}$ Thus, a variety of integrins, especially those of the $\beta 1$ family, have been found to be overexpressed in RA SFs, ${ }^{5}$ and it has been shown that blocking these integrins on the surface of RA SFs reduces their attachment and invasive capacity. ${ }^{67}$ In addition to integrins, transmembrane heparan sulfate proteoglycans of the syndecan family are involved in the attachment of cells to matrix components and there is evidence for a specific contribution of syndecan-4 in the activation of SFs. ${ }^{8}$ Nonetheless, the relevance of individual adhesion molecules as well as their specific binding partners in healthy and diseased cartilage has not been clarified, and recent data ${ }^{9}$ have complicated the picture by showing no significant differences in the overall attachment behaviour between RA SFs and osteoarthritis SFs.

In this study we used the human tumour necrosis factor (TNF) transgenic (hTNFtg) mouse model of 
RA to study the in vivo evolution of cartilage damage and synovial attachment to cartilage and performed in vitro attachment analyses to assess the specific contribution of matrix changes for the initiation and perpetuation of cartilage destruction.

\section{METHODS \\ Mice}

Mice heterozygous for the transgene of human soluble TNF (hTNFtg, strain Tg197; genetic background C57BL/6) were originally generated and kindly provided by Dr Kollias and coworkers. ${ }^{10}$ These mice develop an inflammatory destructive arthritis from the age of 4 weeks onwards due to constitutive overexpression of human TNF. ${ }^{11} 12$ We analysed the time course of histological changes in these animals, and also evaluated the contribution of interleukin (IL)-1 to the synovial attachment to cartilage. Based on data suggesting an impact of IL-1 deficiency in TNF-mediated joint destruction, ${ }^{13}$ we generated hTNTFtg/ IL-1 $1^{-/}$animals by crossing hTNFtg animals with IL- $1 \mathrm{a} / \mathrm{b}^{-/-}$mice (C57Bl/6) kindly provided by Dr Iwakura. ${ }^{14}$

Since transmembrane heparan sulfate proteoglycans of the syndecan family were identified as important regulators of cell attachment to matrix components, ${ }^{8}$ we also assessed the effects of syndecan-4 on SF behaviour and attachment in inflammatory arthritis by crossing hTNFtg mice with syndecan- $4^{-/}$mice (C57B1/6) kindly provided by Dr Echtermeyer ${ }^{15}$ to generate hTNFtg/syn-4 $4^{-/-}$mice.

All resulting genotypes of the respective F2 generation were comparatively analysed. Appropriate wild type (wt) C57B1/6 littermates served as controls for all experiments.

\section{Histomorphometric analysis}

Hind paws of hTNFtg mice at different ages, namely 2 ( $n=6), 4$ $(n=10), 6(n=6), 8(n=6), 10(n=7), 12(n=7)$ and $14(n=6)$ weeks, were fixed overnight in $4.0 \%$ formalin and then decalcified in 14\% EDTA (Sigma-Aldrich, St Louis, Missouri, USA). Paraffin sections $(3 \mu \mathrm{m})$ of hind paws were stained with toluidine blue for the assessment of synovial inflammation, cartilage degradation, attachment of SFs and subchondral bone erosions. To validate the data obtained for cartilage proteoglycan loss with toluidine blue, a comparative staining of paraffin-embedded hind paws of wild type and hTNFtg mice with Safranine-O-fast green was performed at two different time points (early and late stage of disease). For assessment of synovial inflammation, scores of the areas of synovitis in all tarsal joints in each mouse were calculated and expressed as a percentage of the total tissue area.

For cartilage degradation analyses we qualitatively determined the amount of toluidine blue-stained cartilage, as healthy cartilage shows a dark blue stain and is destained upon loss of matrix. ${ }^{16}$ To quantify proteoglycan loss, the amount of destained cartilage was related (expressed as a percentage) to dark bluestained cartilage. In the comparative analysis with Safranine-Ofast green staining, assessment was performed accordingly by determining the light red and dark red areas.

Attachment of inflamed synovial tissue to the cartilage surface was quantified by measuring the length of cells adhering to the cartilage (in $\mu \mathrm{m}$ ). The length of subchondral bone erosions was determined in the same way.

Toluidine blue paraffin-embedded sections ( $3 \mu \mathrm{m})$ from hind paws of wt littermates at weeks $8(n=6)$ and $14(n=6)$ served a



week 10

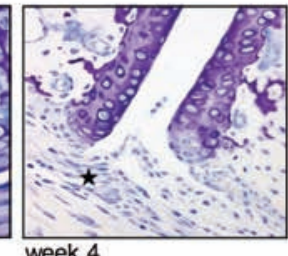
week 4

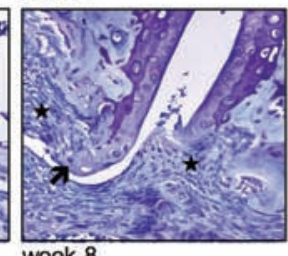

C

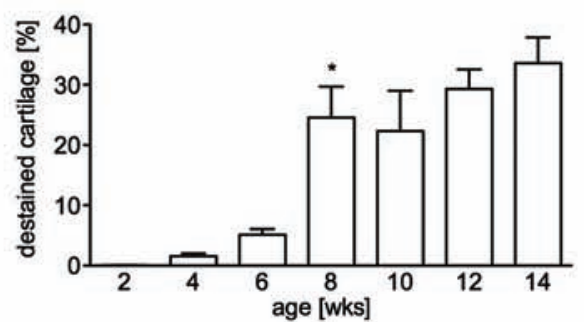

d

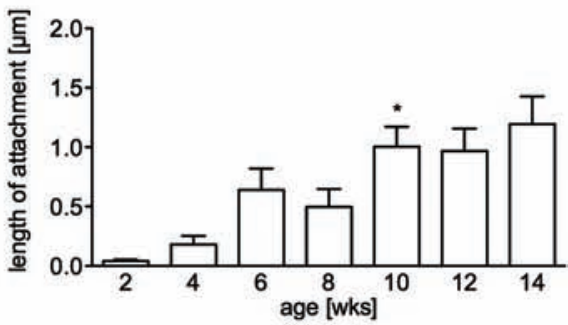

e

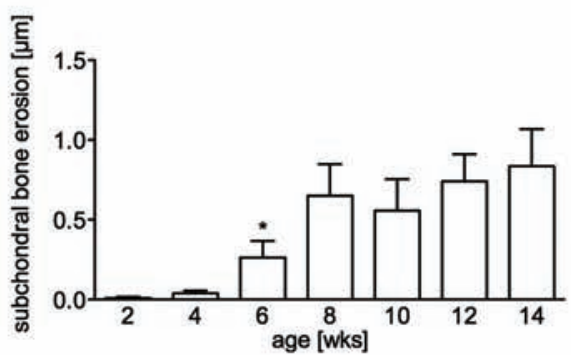

Figure 1 Time course of histological changes in hTNFtg mice. (A) While the synovium and cartilage are intact at week 2, there is a marked increase in synovitis (asterisks) between weeks 4 and 6 . At week 4, signs of cartilage destaining are detectable (red arrow) without attachment of the synovial tissue. As the disease progresses (weeks 8-14), a prominent invasion by the pannus at the junctional zone between cartilage and bone leads to the destruction of bone and mineralised cartilage. (B-E) Histomorphometric analysis showed that the inflamed synovial tissue develops from week 4 and then increases significantly between weeks 4 and 6 . While first signs of destaining indicating a loss of proteoglycans can be found earlier, a relevant loss of proteoglycans occurs only around week 8 . About 2 weeks after proteoglycan loss peaks, increased synovial tissue attachment (light arrows) to cartilage becomes significant; subchondral bone is a primary target of inflammatory joint destruction and increases steadily from week 6 (mean \pm SEM, $\left.{ }^{*} \mathrm{p}<0.05, \mathrm{n}=6\right)$. 
as controls. Sections from hTNTFtg/IL-1 ${ }^{-/-}(\mathrm{n}=6)$ and hTNFtg/ syn- $4^{-1-}(\mathrm{n}=6)$ mice were analysed at the age of 8,14 and 16 weeks, respectively, to quantify the effects of IL-1 or syndecan-4 deficiency on TNF-mediated inflammatory arthritis.

All analyses were performed by one blinded observer (AK) using a Zeiss Axioskop 2 microscope (Carl Zeiss, Marburg, Germany) equipped with a digital camera and image analysis system (Osteomeasure, OsteoMetrics, Decatur, Georgia, USA).

\section{Immunohistochemistry}

For immunohistochemical analyses, sections were stained with antibodies to IL-1 (1:25 dilution; R\&D Systems, Minneapolis, Minnesota, USA) and IL-6 (1:25 dilution; Acris Antibodies, Hiddenhausen, Germany). Sections were pretreated for $20 \mathrm{~min}$ at $96^{\circ} \mathrm{C}$ and blocking of endogenous peroxidase was done with $0.3-3 \%$ hydrogen peroxide in phosphate buffered saline (PBS) for $10 \mathrm{~min}$.

\section{In vitro attachment assay}

To study the effects of proteoglycan loss on the attachment of SFs in vitro, an attachment assay with freshly isolated murine cartilage explants and isolated SFs was used. ${ }^{17}$ To this end, hip joints of wt and hTNFtg mice were opened after the animals were killed at the age of 4, 8 and 12 weeks ( $n=4$ in each group) and cartilage of the femoral head ('cartilage cap') was obtained aseptically. Human femoral heads were obtained from three patients undergoing trauma surgery and cartilage was removed from bone. Cartilage tissue was then cultivated in Dulbecco's Modified Eagle's Medium (DMEM) high glucose containing $10 \%$ fetal calf serum, ab/am, $10 \mathrm{mM}$ HEPES and $50 \mu \mathrm{g} / 500 \mathrm{ml}$ ascorbic acid for $24 \mathrm{~h}$ at $37^{\circ} \mathrm{C}, 5 \% \mathrm{CO}_{2}$. Subsequently, to induce proteoglycan loss, recombinant IL-1 $\beta$ (R\&D, Minneapolis, Minnesota, USA) was added at concentrations of $1 \mathrm{ng} / \mathrm{ml}$ and $10 \mathrm{ng} / \mathrm{ml}$ for $24 \mathrm{~h}$. Furthermore, cartilage was incubated with collagenase (Worthington, Lakewood, New Jersey, USA) at a concentration of $0.25 \mathrm{mg} / \mathrm{ml}^{18}$ for $24 \mathrm{~h}$. To exclude continuing IL-1 or collagenase effects, cartilage was washed gently three times with PBS after harvesting the supernatants for analysis of sulphated glycosaminoglycan (sGAG) content using an Alcian blue binding assay (Wieslab AB, Lund, Sweden) as described previously. ${ }^{17}$

SFs were isolated from the hind paws of wt $(n=6)$, hTNFtg $(n=6)$ and hTNFtg/syn- $4^{-/-}(n=5)$ mice and human synovial tissue (obtained from three patients with RA during planned joint surgery with dermal fibroblasts serving as controls) by enzymatic digestion as described previously. ${ }^{19}$ Cells were used between passage four and six. For attachment analyses, $6 \times 10^{4}$ SFs of all genotypes were seeded onto cartilage for $2 \mathrm{~h}$ under continuous rotation. Cartilage and cells were then incubated for $12 \mathrm{~h}$ in high glucose-containing DMEM. Finally, cartilage and attached cells were fixed in $4.0 \%$ formalin and stained with haematoxylin $(n=4)$. For quantification by light microscopy, the numbers of attached SFs were counted manually across the whole cartilage surface.

\section{Statistical analysis}

Data are expressed as mean \pm SEM. Differences between different time points were compared using the Mann-Whitney $\mathrm{U}$ test. The Mann-Whitney $\mathrm{U}$ test was also used to compare differences between wt, hTNFtg and hTNFtg/IL-1-1- and hTNFtg/syn- $4^{-/-}$mice. A p value of $\leq 0.05$ was considered as significant.

\section{RESULTS}

Loss of cartilage proteoglycans precedes the attachment and invasion of inflamed synovial tissue in hTNFtg animals

We first analysed the time course of histological changes in the inflamed joints of hTNFtg mice (figure 1A). At week 2 we did not detect synovitis or cartilage destruction. However, at week 4 we saw the first signs of synovial inflammation which had continuously increased by week 6 . The amount of inflamed synovial tissue increased until week 14 (figure 1B).

In parallel to the first signs of synovial inflammation at week 4, we also detected the first evidence of cartilage damagenamely, a loss of cartilage proteoglycans as indicated by cartilage destaining. However, in contrast to synovial inflammation, the strongest increase in cartilage damage occurred between weeks 6 and 8 when the percentage of destained cartilage increased significantly from $5 \%$ to $25 \%$ ( $p<0.05)$. From this point on, loss of cartilage proteoglycans progressed slightly, reaching its maximum of $34 \%$ at week 14 (figure 1C). The comparative analysis of proteoglycan loss using Safranine-O-fast green staining showed similar results (see figure S1A,B in online supplement).

Attachment of synovial tissue to the cartilage did not precede but followed cartilage degradation by about 2 weeks. We found a minimal attachment to the articular cartilage after week 4 (0.2 $\mu \mathrm{m})$. However, the most prominent increase in synovial attachment occurred between weeks 8 and 10 (from $0.5 \mu \mathrm{m}$ at week 8 to $1.21 \mu \mathrm{m}$ at week $10, \mathrm{p}<0.05)$, subsequent to the severe proteoglycan loss that had occurred between weeks 6 and 8 (figure 1D). We also found a prominent increase of subchondral bone erosions between weeks 4 and 6 (from $0.03 \mu \mathrm{m}$ to $0.3 \mu \mathrm{m}$, $\mathrm{p}<0.05$; figure $1 \mathrm{E})$.

\section{IL-1 induced loss of proteoglycans is required for the attachment of SFs}

Based on the established role of IL-1 in the joint destruction of hTNFtg mice, we next wanted to characterise the expression of IL- 1 and IL- 6 (as another inflammatory mediator) over the time course of the disease. We found an increasing expression of IL-1 in the inflamed synovial tissue of hTNFtg animals over time, starting as early as week 4 (see figure S1E in online supplement) while IL-6 expression was negligible (see figure S1F in online supplement). We therefore investigated whether the loss of IL-1 may also affect the attachment of the synovial tissue to the cartilage in the course of TNF-driven arthritis. Histopathological analyses of hTNFtg and hTNFtg/IL-1 $1^{-/}$mice showed that, in contrast to the hTNFtg animals, only a small amount of destained cartilage was detectable in hTNFtg/IL-1-/- animals (figure $2 \mathrm{~A}$ ). Moreover, in hTNFtg/IL-1 $1^{-/}$animals the attachment of the pannus to the cartilage was reduced compared with hTNFtg animals as early as at week $8(0.12 \mu \mathrm{m}$ vs $0.5 \mu \mathrm{m}, \mathrm{p}<0.05)$ and most significantly at later stages of the disease at week $16(0.15 \mu \mathrm{m}$ vs $1.6 \mu \mathrm{m}, \mathrm{p}<0.05$; figure $2 \mathrm{~B}$ ).

To further differentiate the roles of the two compartments involved in TNF-mediated cartilage destruction-namely, the cartilage on one hand and the inflamed synovial membrane on the other hand-we performed further in vitro analysis.

We analysed the capacity of wt SFs and hTNFtg SFs to attach in vitro to cartilage of 4-, 8- and 12-week-old wt or hTNFtg mice, respectively. Generally, SFs attached poorly to both wt and hTNFtg cartilage obtained from young (ie, 4-week-old) mice. In contrast, while the attachment of wt SFs remained poor when wt cartilage was used from older (ie, 8- and 12-week-old mice), hTNFtg cartilage from 8-week-old mice facilitated the attachment of wt and even more prominently of hTNFtg SF. 


\section{a}
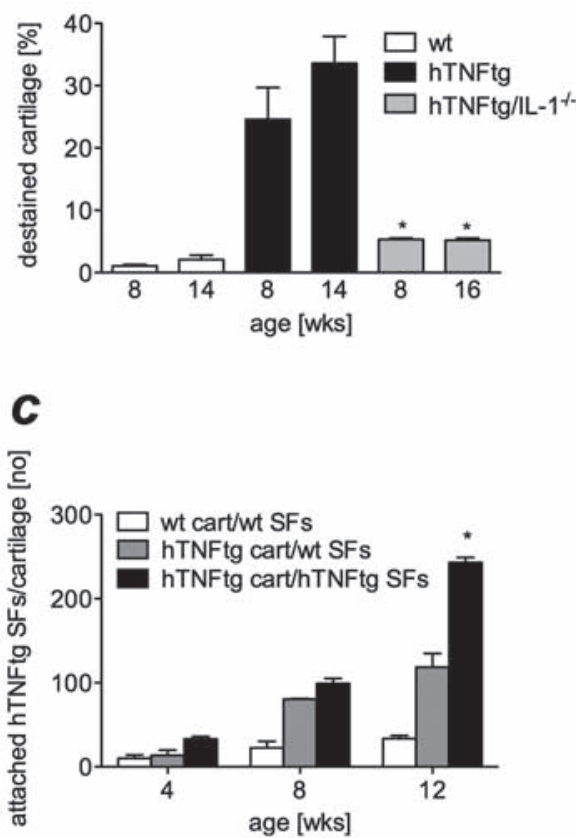

e

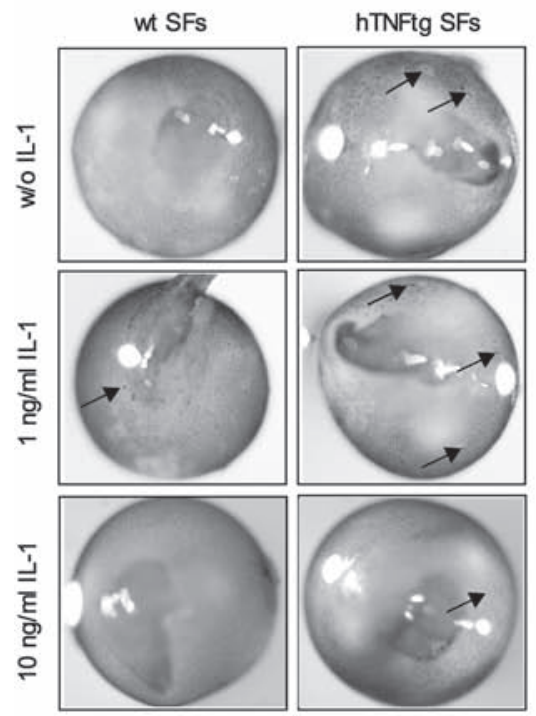

b

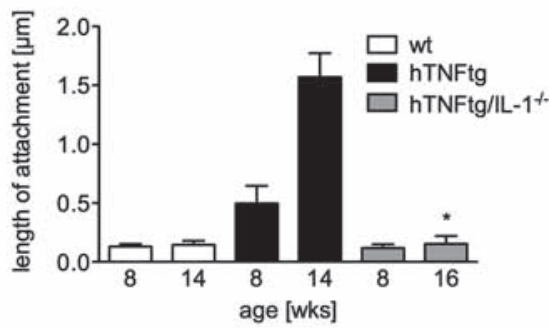

d
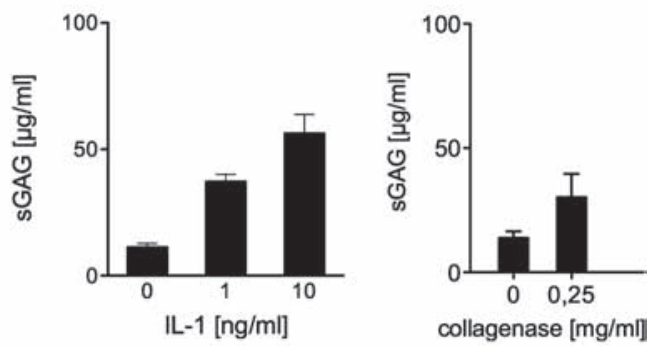

f
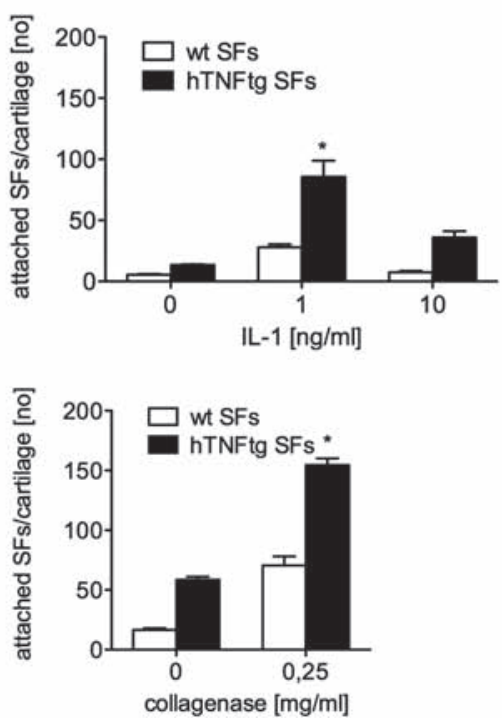

Figure 2 Interleukin (IL)-1 is an important trigger of early proteogylcan loss that elicits synovial cell attachment in vivo and in vitro. $(A, B)$ IL-1 deficiency in hTNFtg mice leads to a significant reduction in proteoglycan loss and prevents the attachment of the inflamed synovial tissue to the articular cartilage in the course of disease (mean $\pm S E M,{ }^{*} p<0.05, n=6$ ). (C) Analyses of wild type and hTNFtg synovial fibroblast (SF) attachment to cartilage in 4-, 8- and 12-week-old wt or hTNFtg mice. At week 4, SFs attached poorly to wt and hTNFtg cartilage, the low levels of attachment persisted when wt cartilage from 8- and 12-weeks-old mice was assessed; in contrast, cartilage from 8-week-old hTNFtg mice facilitated the attachment of wt and especially of hTNFtg SF. Cartilage from 12-week-old hTNFtg mice resulted in a prominent attachment of hTNFtg fibroblasts (mean \pm SEM, ${ }^{*} p<0.05, n=4$ ). (D) Incubation of hTNFtg cartilage explants with different concentrations of IL-1 and collagenase leads to a dosedependent loss of proteoglycans (mean $\pm S E M,{ }^{*} p<0.05, n=6$ ). (E, F) As shown by representative images $(E)$ and upon quantification $(F)$, wt or hTNFtg SFs hardly attach to intact untreated cartilage explants. When cartilage explants are pretreated with $1 \mathrm{ng} / \mathrm{ml}$ IL-1, SFs especially from hTNFtg mice exhibit a pronounced attachment ( $\mathrm{E}$, arrows). Similar findings can also be observed with collagenase treatment. A higher concentration of IL-1 $(10 \mathrm{ng} / \mathrm{ml})$ did not further increase but rather reduced the attachment of wt and hTNFtg SFs (mean \pm SEM, ${ }^{*} \mathrm{p}<0.05, \mathrm{n}=4$ ).

Cartilage of 12-week-old hTNFtg mice slightly increased the attachment of wt SFs but resulted in a more prominent attachment of hTNFtg SFs, which was nearly twice as high as that of wt cells (figure $2 \mathrm{C}$ ).

We next investigated if the loss of proteoglycans induced by inflammatory cytokines such as IL-1 may be a prerequisite for the attachment of SFs to cartilage. To this end, we first incubated cartilage from 4-week-old mice with IL-1 and found a dose-dependent release of proteoglycans from the cartilage (figure 2D). We next investigated the attachment of SFs from wt and hTNFtg mice to 4-week-old cartilage derived from wt mice. Loss of proteoglycans as induced by $1 \mathrm{ng} / \mathrm{ml}$ of IL-1 strongly facilitated SF 

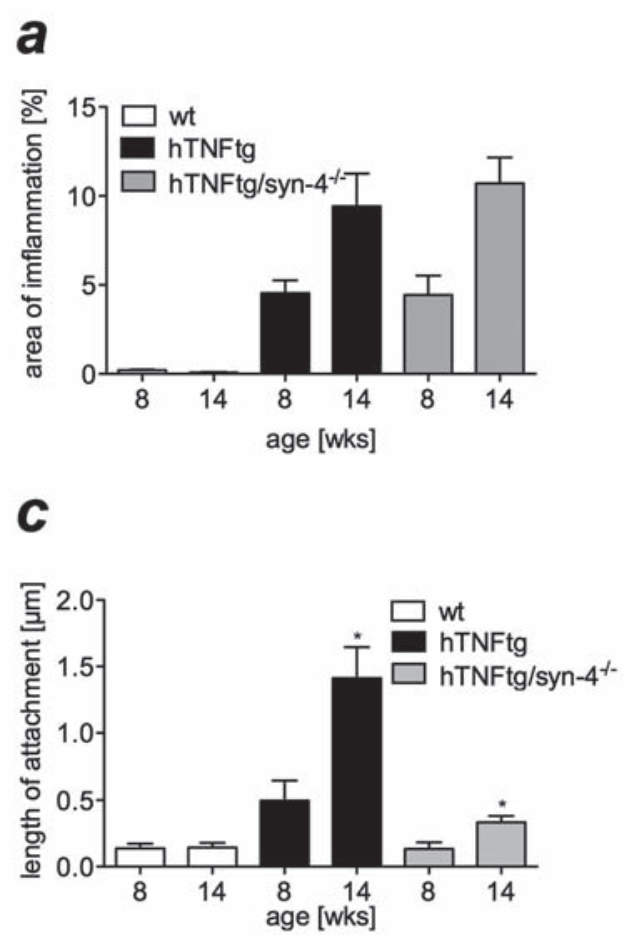

e b

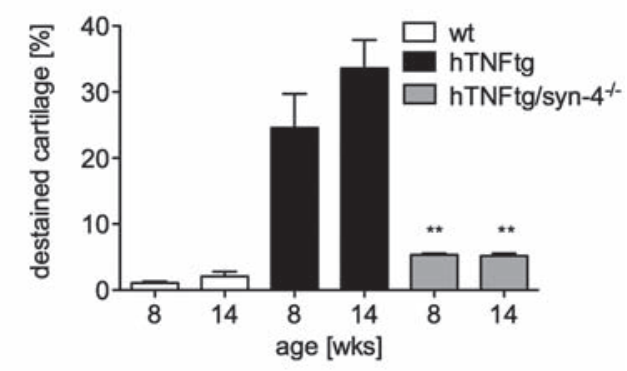

d

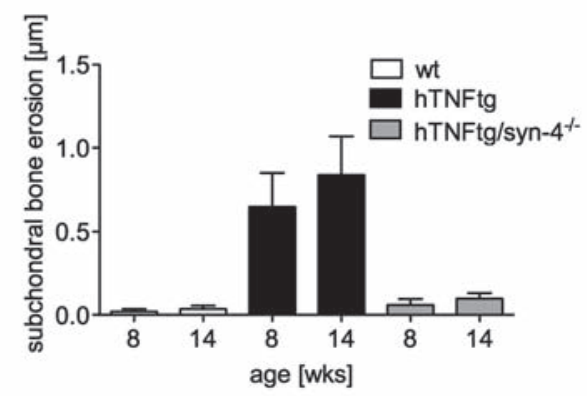

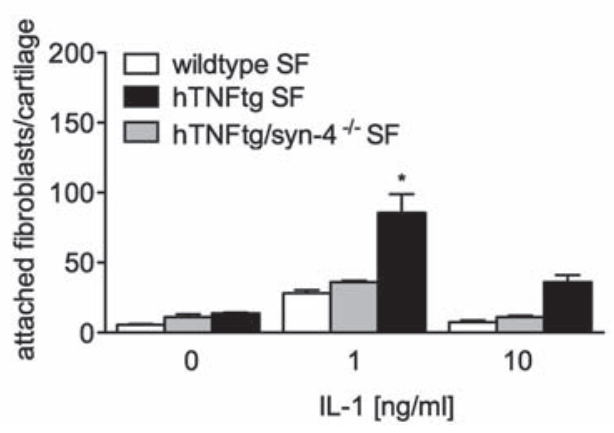

Figure 3 Syndecan-4 mediates the attachment of synovial fibroblasts (SFs) to damaged cartilage. Analyses of histopathological changes of wt, hTNFtg and hTNFtg/syn $4^{-1-}$ mice were performed at weeks 8 and 14. (A) hTNFtg and hTNFtg/syn4 ${ }^{-1-}$ mice show comparable levels of synovial inflammation at both time points (mean $\pm S E M, n=6$ ). (B) A significant reduction of cartilage destaining in $\mathrm{hTNFtg} / \mathrm{syn}-4^{-/-}$mice compared with hTNFtg mice was observed at weeks 8 and $14\left({ }^{* *} p<0.01, n=6\right)$. (C) The attachment of inflamed synovial membrane to the articular cartilage was significantly reduced in hTNFtg/syn4 ${ }^{-/}$mice compared with hTNFtg animals at week 14 (mean \pm SEM, ${ }^{*} \mathrm{p}<0.05, \mathrm{n}=6$ ). (D) hTNFtg/syn- $4^{-/-}$mice show noticeably less subchondral bone erosions than hTNFtg animals at both time points (mean \pm SEM, $n=6$ ). (E) In vitro, SFs from $\mathrm{hTNFtg} / \mathrm{syn}^{-4^{-1-}}$ mice showed a significantly reduced attachment to interleukin (IL)-1 pretreated cartilage compared with hTNFtg fibroblasts (mean $\left.\pm S E M,{ }^{*} p<0.05, n=4\right)$.

attachment to cartilage. Of note, attachment of hTNFtg SFs was significantly higher than that of wt SFs. $10 \mathrm{ng} / \mathrm{ml}$ of IL-1 resulted in an overall lesser attachment of SFs compared with $1 \mathrm{ng} / \mathrm{ml}$, but hTNFtg SFs still exhibited a stronger adhesion than did wt SFs (figure 2E,F). There was very little attachment of either wt or hTNFtg SFs to untreated cartilage (figure 2F).

To further elucidate if proteoglycan loss elicited by IL-1 or other IL-1-mediated effects are essential for the increased attachment of SFs to cartilage, we induced proteoglycan degradation independent of IL-1 by addition of collagenase (figure 2D). We found that, similar to the effects of IL-1, collagenase-induced cartilage damage strongly facilitated SF attachment to cartilage, and this effect was more pronounced when hTNFtg SFs were studied (figure 2F). These data suggest that loss of cartilage matrix is a prerequisite to synovial attachment.

To determine if these mechanisms are also of relevance in human RA, we analysed the attachment of RA SFs and control dermal fibroblasts to human cartilage after treatment with IL-1 or collagenase, respectively. Dermal fibroblasts hardly attached to untreated human cartilage and treatment of cartilage with $1 \mathrm{ng} / \mathrm{ml} \mathrm{IL-1,} \mathrm{but} \mathrm{not} \mathrm{with} \mathrm{collagenase,} \mathrm{only} \mathrm{slightly} \mathrm{increased}$ their attachment (see figure S1C,D in online supplement). In contrast, SFs from patients with RA showed significant attachment to cartilage pretreated with either $1 \mathrm{ng} / \mathrm{ml}$ IL-1 or collagenase. These data confirm that proteoglycan loss also facilitates the attachment of SFs to cartilage in humans.

\section{Attachment of SFs is mediated by syndecan-4}

Based on the notion that, in addition to integrins, transmembrane heparan sulfate proteoglycans of the syndecan family are critically involved in the attachment of cells to matrix components, ${ }^{8}$ we examined whether lack of syndecan-4 affects cartilage degradation and destruction in the hTNFtg mouse model. Analysis of histopathological changes in hTNFtg/syn- $4^{-/-}$mice showed 
no differences in the amount of synovial inflammation compared with hTNFtg animals (figure 3A). However, there was a significant reduction in cartilage destaining in hTNFtg/syn- $4^{-/-}$ mice at week 8 (5\% vs $25 \%, \mathrm{p}<0.01)$ and week 14 (5\% vs $34 \%$, $\mathrm{p}<0.01$; figure $3 \mathrm{~B})$. Furthermore, 8-week-old hTNFtg/syn- $4^{-/-}$ animals exhibited only poor synovial attachment $(0.11 \mu \mathrm{m}$ vs $0.52 \mu \mathrm{m})$ and even 14-week-old animals showed less attachment than hTNFtg mice early in the course of the disease at week 8 $(0.32 \mu \mathrm{m}$ vs $1.6 \mu \mathrm{m}, \mathrm{p}<0.05$; figure $3 \mathrm{C})$. In addition, the extent of subchondral bone erosions was also clearly reduced in hTNFtg/ syn- $4^{-/-}$mice compared with hTNFtg mice (week 8; $0.06 \mu \mathrm{m}$ vs $0.7 \mu \mathrm{m}$; week 14: $0.1 \mu \mathrm{m}$ vs $0.84 \mu \mathrm{m}$; figure $3 \mathrm{D})$.

In line with these data, hTNFtg/syn- $4^{-/-}$SFs differed from hTNFtg SFs by showing significantly less attachment to IL-1treated cartilage explants from wt mice in vitro (figure $3 \mathrm{E}$ ).

\section{DISCUSSION}

Joint destruction is a key feature of RA and can result in severe disability in many affected individuals. In this context, the loss of articular cartilage is of particular importance because it is largely irreversible and thus constitutes a 'point of no return' in the destruction of RA joints. While it has been widely accepted that inflammatory stimuli such as TNF $\alpha$ lead to synovial fibroblast activation resulting in the induction and upregulated expression of adhesion molecules such as integrins (eg, $\alpha 2 \beta 1$ integrin) ${ }^{20}$ and syndecan- $4^{21}$ and matrix degrading enzymes, the direct relationship between cartilage damage and SF activation has been less well defined. While there are a number of inflammatory joint diseases that, despite severe chronic synovitis, rarely result in cartilage damage, joint destruction in RA has been demonstrated to occur or proceed even when synovial inflammation is well controlled. These observations raise the question about the peculiarities of synovial inflammation in RA and, even more strikingly, about the specific relationship between alterations in the local (ie, cartilage) environment and the attachment and invasion of the inflamed synovial tissue. Recent data indicate that cytokines present in the RA synovial membrane trigger the destructive process largely independently of other aspects of the synovial pathology, since the deficiency of IL-1 in hTNFtg mice does not affect the inflammatory aspects of synovitis but prevents cartilage and reduces bony destruction. ${ }^{132}$

Based on these data, we hypothesised that the early loss of cartilage components elicited by the release of various factors is an important prerequisite for the attachment of inflamed synovial tissue to the cartilage and thus for subsequent irreversible cartilage damage. To address this question we used the hTNFtg mouse model of RA because it is characterised by a spontaneous destructive arthritis resembling human RA, particularly with respect to cartilage and bone destruction. ${ }^{10}$

Analysis of the arthritic changes in these mice revealed a sequence of events with inflammation occurring first, closely followed by loss of proteoglycans from the articular cartilage. These data indicate that, in hTNFtg animals, loss of articular proteoglycans is induced by synovial inflammation but clearly precedes direct attachment and subsequent invasion of inflamed and hyperplastic synovial tissue into the cartilage matrix. Interestingly, loss of proteoglycans did not appear to require direct contact between the inflamed synovial tissue and the cartilage. Since, in the absence of undermining bone erosions, cartilage matrix degradation only occurs in its synoviotropic portions, we speculated that molecules released from the inflamed synovial membrane lead to the observed loss of cartilage matrix. As the attachment of SFs to the cartilage reached its maximum just 2 weeks after the initial peak in proteoglycan loss, it seems that mechanisms associated with the early loss of cartilage matrix are an important prerequisite for the attachment. Interestingly, invasion into the cartilage is not a primary event in the hTNFtg model of RA, suggesting that such invasion in the human disease may be a consequence of TNF-independent mechanisms.

The results prompted us to analyse more specifically the role of IL-1, as it has been emphasised that IL-1 might be superior to TNF in facilitating the attachment to cartilage. ${ }^{23}$ Moreover, IL-1 has been strongly associated with the loss of cartilage proteoglycans in different animal models of human RA, such as zymosan-induced arthritis, ${ }^{24}$ antigen-induced arthritis, ${ }^{24}$ collagen-induced arthritis, ${ }^{22} 25$ streptococcal cell wall arthritis ${ }^{22} 25$ and the hTNFtg animal model used in this study. ${ }^{13} 26$ Moreover, overexpression of IL-1 by gene transfer in rabbit knee joints has been shown to produce key features of human RA including cartilage damage. ${ }^{27}$

In line with these data, and especially with the aforementioned observations in hTNFtg mice, ${ }^{13}$ we found that hTNFtg/ IL $-1^{-/}$mice are protected from cartilage damage, particularly the early loss of proteoglycans, and also exhibit only very limited attachment of the inflamed synovial tissue to the cartilage which, despite the differences in synovitis, hardly differs from that of wt mice. To further substantiate the hypothesis that inflammatory (particularly IL-1-induced) cartilage damage is required for the attachment of synovial cells, we used an in vitro attachment assay by seeding SFs from hTNFtg or wt mice onto freshly isolated articular cartilage from wt or hTNFtg mice. Of note, SFs from hTNFtg mice hardly attached to wt cartilage, independent of the age of the cartilage at the time of explantation. However, hTNFtg SFs attached to cartilage explants from diseased hTNFtg mice and this attachment increased with the age of the mice at the time of cartilage explantation-that is, with damage to the hTNFtg cartilage prior to explantation. These data support our in vivo observations and indicate that SFs hardly attach to intact cartilage and that mechanisms leading to the loss of cartilage matrix components such as proteoglycans constitute an important step in the induction of attachment. This notion is further supported by the observation that both wt and hTNFtg SFs showed only negligible attachment to untreated cartilage. However, cartilage explants from wt mice treated with IL-1 have a higher susceptibility to attachment by both wt and hTNFtg SFs in vitro, supporting the role of IL-1-induced cartilage damage in the pathogenesis of RA. It is important to mention that previous data had indicated that IL-1-independent mechanisms might also lead to cartilage damage in patients with RA as SFs transfected with IL-1R antagonist are still able to attach and invade into the cartilage. ${ }^{28}$ The finding of a decreasing attachment of SFs with increasing doses of IL-1 despite increasing proteoglycan loss might also point to the involvement of additional mechanisms. Moreover, the fact that hTNFtg SFs have a greater capacity to bind to either wt or hTNFtg cartilage points also to effects on SFs independent of the condition of the cartilage. It may also be speculated that IL-1 plays a more prominent role in the hTNFtg model than in human RA while IL-6, for example, is less important, as suggested by our immunohistochemical stainings for IL-6. This notion is compatible with recent data showing that IL-6R blockade, though reducing osteoclast formation, did not inhibit synovial inflammation in hTNFtg mice. ${ }^{29}$ Our data suggest that loss of proteoglycans or proteoglycan fragments facilitates the attachment of SFs and that, later in the course of disease, attachment does not increase further. We have shown that a candidate molecule (ie, syndecan-4) is critically involved in SF attachment to IL-1 damaged cartilage in vitro and in vivo, and it may be possible to narrow down the list of potential 


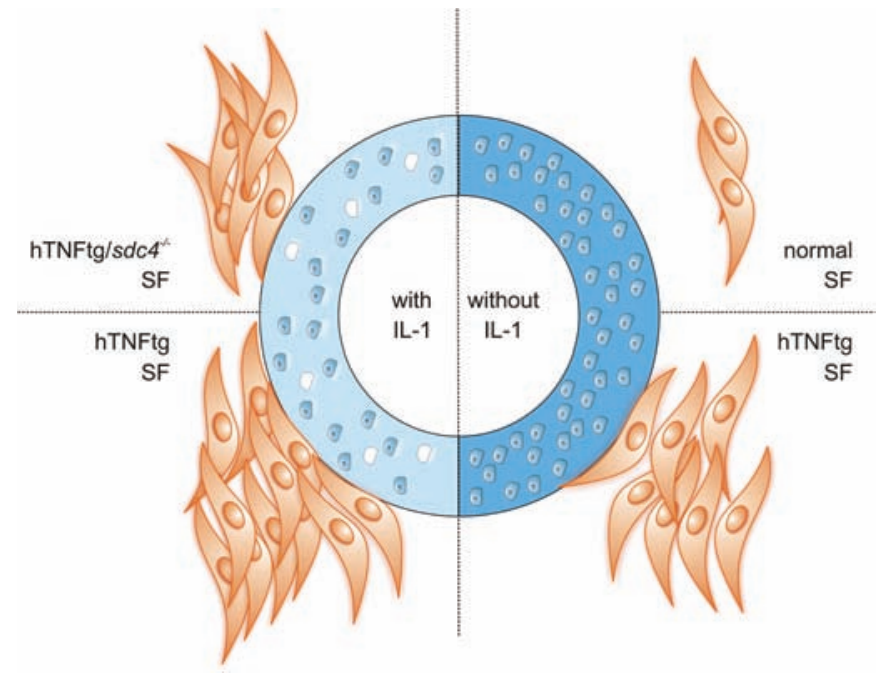

Figure 4 Regulation of fibroblast attachment by interleukin (IL)-1mediated loss of cartilage damage. Healthy articular cartilage (right) provides no substrate for healthy wt fibroblasts and also hTNFtg synovial fibroblasts (SFs) show only limited attachment to intact cartilage. The loss of proteoglycans as induced by IL-1 (left) greatly facilitates the attachment of SFs, particularly of hTNFtg fibroblasts. This attachment to as yet undefined proteoglycans or proteoglycan fragments is mediated also by syndecan- 4 because the loss of syndecan-4 decreases the attachment of SFs to IL-1-treated cartilage.

candidates which, aside from syndecan-4, comprise fibronectin or fibronectin fragments that have been reported to be released from the cartilage in RA and to stimulate SFs. ${ }^{30}{ }^{31}$ In this context it needs to be emphasised that, while binding of components of the extracellular matrix such as fibronectin to syndecan-4 has been well established, the observation that the loss of syndecan-4 leads to an altered attachment of the inflamed synovial tissue and of arthritic SFs to cartilage is novel and has not been reported previously. Interfering with syndecan-4-mediated cell attachment may therefore constitute a novel approach for treating cartilage damage in RA.

In summary, our data provide evidence that loss of cartilage proteoglycans is an early event in the cascade of cartilage damage in the hTNFtg mouse model of RA. This loss of proteoglycans is induced by cytokines such as IL-1, precedes the attachment and invasion of the inflamed synovial tissue into cartilage in vivo and constitutes an important prerequisite for inflamed synovial tissue attachment and subsequent cartilage destruction (figure 4). While the exact nature of the matrix molecules that are responsible for this attachment remains to be determined, it appears that, in addition to integrins, syndecan-4 is involved prominently in cartilage degradation and fibroblast attachment. Interestingly, synovial inflammation is unaffected, which is in line with the phenotype of hTNFtg/ IL-1 ${ }^{-/-}$mice. ${ }^{13}$

It therefore appears that cartilage damage in RA is initiated by the presence of soluble molecules released from the inflamed synovial tissue such as cytokines and matrix degrading enzymes, followed by synovial attachment which subsequently leads to cartilage destruction. The progression of cartilage damage even in patients whose disease activity has been controlled may reflect the complexity of these events. Importantly, however, the data presented suggest several new therapeutic targets for prevention of cartilage damage and thus the irreversible disability in RA.
Acknowledgements The authors thank Yoichiro Iwakura for providing the IL-1a/b $\mathrm{b}^{-/-}$mice and Georg Kollias for providing the hTNFtg animals.

Funding This project was supported by Coordination Theme 1 (Health) of the European Community's FP7, grant agreement number HEALTH-F2-2008-223404 (MASTERSWITCH) and the Deutsche Forschungsgemeinschaft (DFG), grant number Pa689/7-1 and Pa689/7-2.

Competing interests None.

Ethics approval All animal procedures were approved by the local ethics committee.

Provenance and peer review Not commissioned; externally peer reviewed.

\section{REFERENCES}

1. Scott DL, Pugner K, Kaarela K, et al. The links between joint damage and disability in rheumatoid arthritis. Rheumatology (Oxford) 2000;39:122-32.

2 Karouzakis E, Neidhart M, Gay RE, et al. Molecular and cellular basis of rheumatoid joint destruction. Immunol Lett 2006;106:8-13.

3. Ishikawa H, Ohno 0, Saura R, et al. Cytokine enhancement of monocyte/synovial cell attachment to the surface of cartilage: a possible trigger of pannus formation in arthritis. Rheumatol Int 1991;11:31-6.

4. Müller-Ladner U, Gay RE, Gay S. Molecular biology of cartilage and bone destruction. Curr Opin Rheumatol 1998;10:212-19.

5. Ishikawa $\mathbf{H}$, Hirata $\mathbf{S}$, Andoh $\mathrm{Y}$, et al. An immunohistochemical and immunoelectron microscopic study of adhesion molecules in synovial pannus formation in rheumatoid arthritis. Rheumatol Int 1996; 16:53-60.

6. Wang AZ, Wang JC, Fisher GW, et al. Interleukin-1 beta-stimulated invasion of articular cartilage by rheumatoid synovial fibroblasts is inhibited by antibodies to specific integrin receptors and by collagenase inhibitors. Arthritis Rheum 1997:40:1298-307.

7. Sarkissian M, Lafyatis R. Integrin engagement regulates proliferation and collagenase expression of rheumatoid synovial fibroblasts. J Immunol 1999;162:1772-9

8. Echtermeyer $\mathbf{F}$, Bertrand J, Dreier R, et al. Syndecan-4 regulates ADAMTS-5 activation and cartilage breakdown in osteoarthritis. Nat Med 2009;15:1072-6.

9. Schedel J, Wenglén C, Distler 0, et al. Differential adherence of osteoarthritis and rheumatoid arthritis synovial fibroblasts to cartilage and bone matrix proteins and its implication for osteoarthritis pathogenesis. Scand J Immunol 2004; 60:514-23.

10. Keffer J, Probert L, Cazlaris $\mathrm{H}$, et al. Transgenic mice expressing human tumour necrosis factor: a predictive genetic model of arthritis. EMBO J 1991;10:4025-31.

11. Hayer S, Redlich K, Korb A, et al. Tenosynovitis and osteoclast formation as the initial preclinical changes in a murine model of inflammatory arthritis. Arthritis Rheum 2007; 56:79-88.

12. Blüml S, Binder NB, Niederreiter B, et al. Antiinflammatory effects of tumor necrosis factor on hematopoietic cells in a murine model of erosive arthritis. Arthritis Rheum 2010;62:1608-19.

13. Zwerina J, Redlich K, Polzer K, et al. TNF-induced structural joint damage is mediated by IL-1. Proc Natl Acad Sci USA 2007:104:11742-7.

14. Horai R, Asano M, Sudo K, et al. Production of mice deficient in genes for interleukin (IL)-1alpha, IL-1 beta, IL-1alpha/beta, and IL-1 receptor antagonist shows that IL-1beta is crucial in turpentine-induced fever development and glucocorticoid secretion. J Exp Med 1998;187:1463-75.

15. Echtermeyer F, Streit M, Wilcox-Adelman S, et al. Delayed wound repair and impaired angiogenesis in mice lacking syndecan-4. J Clin Invest 2001;107:R9-R14.

16. Blumenfeld I, Gaspar R, Laufer D, et al. Enhancement of toluidine blue staining by transforming growth factor-beta, insulin-like growth factor and growth hormone in the temporomandibular joint of aged mice. Cells Tissues Organs (Print) 2000;167:121-9.

17. Liu G, Kawaguchi H, Ogasawara T, et al. Optimal combination of soluble factors for tissue engineering of permanent cartilage from cultured human chondrocytes. J Biol Chem 2007;282:20407-15.

18. Hajiioannou JK, Nikolidakis A, Naumidi I, et al. In vitro enzymatic treatment and carbon dioxide laser beam irradiation of morphologic cartilage specimens. Arch Otolaryngol Head Neck Surg 2006;132:1363-70.

19. Meinecke I, Cinski A, Baier A, et al. Modification of nuclear PML protein by SUMO-1 regulates Fas-induced apoptosis in rheumatoid arthritis synovial fibroblasts. Proc Natl Acad Sci USA 2007;104:5073-8.

20. Peters MA, Wendholt D, Strietholt S, et al. The loss of integrin $\alpha 2 \beta 1$ suppresses joint inflammation and cartilage destruction in mouse models of rheumatoid arthritis. Arthritis Rheum 2011;(In Press).

21. Wang J, Markova D, Anderson DG, et al. TNF- $\alpha$ and IL-1 $1 \beta$ promote a disintegrin-like and metalloprotease with thrombospondin type I motif-5-mediated aggrecan degradation through syndecan-4 in intervertebral disc. J Biol Chem 2011;286:39738-49.

22. Joosten LA, Helsen MM, Saxne T, et al. IL-1 alpha beta blockade prevents cartilage and bone destruction in murine type II collagen-induced arthritis, whereas TNF-alpha blockade only ameliorates joint inflammation. J Immunol 1999;163:5049-55. 
23 Pap T, van der Laan WH, Aupperle KR, et al. Modulation of fibroblast-mediated cartilage degradation by articular chondrocytes in rheumatoid arthritis. Arthritis Rheum 2000; $43: 2531-6$.

24. van de Loo FA, Joosten LA, van Pent PL, et al. Role of interleukin-1, tumor necrosis factor alpha, and interleukin-6 in cartilage proteoglycan metabolism and destruction. Effect of in situ blocking in murine antigen- and zymosan-induced arthritis Arthritis Rheum 1995;38:164-72.

25. Bakker AC, Joosten LA, Arntz 0J, et al. Prevention of murine collagen-induced arthritis in the knee and ipsilateral paw by local expression of human interleukin-1 receptor antagonist protein in the knee. Arthritis Rheum 1997;40:893-900.

26. Polzer K, Joosten L, Gasser J, et al. Interleukin-1 is essential for systemic inflammatory bone loss. Ann Rheum Dis 2010;69:284-90.

27. Ghivizzani SC, Kang R, Georgescu HI, et al. Constitutive intra-articular expression of human IL-1 beta following gene transfer to rabbit synovium produces all major pathologies of human rheumatoid arthritis. J Immunol 1997;159:3604-12.
28. Müller-Ladner U, Roberts CR. Franklin BN, et al. Human IL-1Ra gene transfer into human synovial fibroblasts is chondroprotective. J Immunol 1997

158:3492-8.

29 Axmann R, Böhm C, Krönke G, et al. Inhibition of interleukin-6 receptor directly blocks osteoclast formation in vitro and in vivo. Arthritis Rheum 2009;60:2747-56

30. Homandberg GA, Wen C, Hui F. Cartilage damaging activities of fibronectin fragments derived from cartilage and synovial fluid. Osteoarthritis Cartil 1998:6:231-44.

31. Yasuda T, Poole AR. A fibronectin fragment induces type II collagen degradation by collagenase through an interleukin-1-mediated pathway. Arthritis Rheum 2002;46:138-48.

32. Kuiper $\mathbf{S}$, Joosten LA, Bendele AM, et al. Different roles of tumour necrosis factor alpha and interleukin 1 in murine streptococcal cell wall arthritis. Cytokine 1998; 10:690-702 